\title{
Single Terminal Fault Location by Natural Frequency of Travelling Wave
}

\author{
Zhai Xueming $^{1, a}$, Li Jinze ${ }^{2, b}$ and Wang Jia ${ }^{2, c}$ \\ School of Control and Computer Engineering, North China Electric Power University, Baoding \\ 071003, China. \\ azxm3165@126.com, ${ }^{\mathrm{b}} \mathrm{li}$ jinzel123@163.com, cncepuwangjia@163.com
}

Keywords: natural frequency; global primary natural frequency; ensemble empirical mode WVD algorithm; electricity transmission

\begin{abstract}
The accuracy and reliability of the main components of travelling wave natural frequency is the key of travelling wave in the fault location method based on the natural frequency. To avoid the problem that traditional algorithms rely on a single primary natural frequency are not reliable, a new method considering the global primary natural frequency which is stroked by the fundamental and harmonics is proposed. The calculated fault distance is more accurate and reliable. And the key in the extraction of the natural frequency of the main ingredient adopt the ensemble empirical mode WVD algorithm to improve frequency resolution while effectively suppressing white noise, thus further improving the location accuracy. Simulation experiment by EMTDC show that the method proposed in this paper can get better stability, higher reliability, reduce ambient noise, and the position is not affected by fault distance or other factors.
\end{abstract}

\section{Introduction}

High voltage transmission is the main way of high-capacity, long-distance electricity transmission, therefore, in case of failure and accurate positioning, fast troubleshooting for the safe operation of power system is of great significance. some researchers put forward the line fault location method based on natural frequency ${ }^{[1]}$. Literature ${ }^{[2]}$ proposed that when transmission line fault occurs, there is a certain proportion relationship between frequency components in the fault current and fault distance. We can implement the single positioning by the extraction of the frequency. Accurately extraction of the main natural frequency values is the basis of the travelling wave based on fault location method. When determining the main natural frequency values only rely on the extraction of single main natural frequency of the peak, and the value is affected by noise, discontinuity of lines, line coupling impedance and harmonic interference is very big, the influence of such factors as the solid extraction to advocate natural frequency values cannot be guaranteed, which affects positioning effect.

Considering the problem that the single main natural frequency is not reliable, we proposes a new method that comprehensive consider of fundamental wave and multiple harmonics: calculate the global main frequency, make it to be the weighted average of the main frequency and multiple natural frequency. Using the global main frequency value to locate more accurately.

\section{Electricity Transmission Line Fault Location Method Based on Travelling Wave Natural Frequency}

The Theory of Electricity Transmission Line Fault Location Method Based on Travelling Wave Natural Frequency. Double power transmission line model is shown in figure1, the total length of transmission line is $\mathrm{L}$, both ends of the impedance is $Z_{1}=R_{1}+j \omega L_{1}$ and $Z_{2}=R_{2}+j \omega L_{2}$. Assumes that fault occurs in the distance $\mathrm{d}$ from the 1.

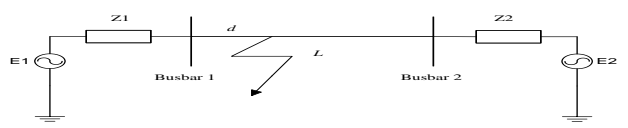

Fig.1 Double-side power supply system 
According to the theory in literature [3], when the line impedance, the relationship between fault distance of the bus 1 side and the natural frequency is:

$$
\begin{aligned}
& d=\frac{\left(\theta_{1}+2 k \pi\right) v}{2 \pi f}, k=0, \pm 1, \pm 2, \ldots \\
& \theta_{1}=\arg \left(\frac{Z_{1}-Z_{2}}{Z_{1}+Z_{2}}\right)
\end{aligned}
$$

$\theta_{1}$ is the travelling wave angle in 1 end; $\mathrm{f}$ is the main natural frequency in 1 end; $\mathrm{v}$ is the corresponding frequency wave velocity; $Z_{1}$ and $Z_{2}$ are impedance at both ends. We can structural equations by formula (1) as follows:

$$
\left\{\begin{array}{c}
d_{1}=\frac{\theta_{1.1} v_{1}}{2 \pi f_{1}} \\
d_{2}=\frac{\left(\theta_{1.2}+2 \pi\right) v_{2}}{2 \pi f_{2}} \\
\vdots \\
d_{n}=\frac{\left(\theta_{1 . n}+2(n-1) \pi\right) v_{n}}{2 \pi f_{n}} \\
\vdots
\end{array}\right.
$$

Formula $\theta_{1.1}$ and $v_{1}$ are the reflection angle and velocity of main natural frequency in 1 side; $\theta_{1.2}$ and $v_{2}$ are the reflection angle and velocity of 2 times main natural frequency; $\theta_{1 . n}$ and $v_{n}$ are the reflection angle and velocity of $n$ times the natural frequency.

The reflection angle is determined by both ends of the line impedance, and when the transmission line is certain, the reflection angle in 1 side can be approximately equal, therefore $\theta_{1.1}=\theta_{1.2}=\ldots=\theta_{1 . n}=\ldots$.

At the same time, according to literature, when traveling wave frequency is high, the wave velocity affected by frequency is small and can be think the same. But the natural frequency is generally in more than a few hundred Hertz, so you can think wave velocity are equal, i.e., the theoretical fault distance obtained by each harmonic is a fixed value too. Will formula (3) in all kinds of additive reduction:

$$
d=\frac{1}{n} \cdot \frac{v_{1.1}}{2 \pi} \cdot\left(\sum_{k=1}^{n} \frac{\theta_{1 . k}+2(k-1) \pi}{f_{k}}+\delta\right), k=1,2, \ldots n
$$

$\delta$ is the residual volume. We treat $\frac{\theta_{1}+\pi+2 k \pi}{f}$ in formula (3) as $f_{\theta}$ which is global main natural frequency. Formula is transformed into:

$$
d=\frac{v f_{\theta}}{2 \pi}
$$

The formula (4) (5) simultaneous reduction available:

$$
f_{\theta}=\frac{1}{n} \cdot\left(\frac{\theta_{1.1}}{f_{1}}+\frac{\theta_{1.1}+2 \pi}{f_{2}}+\cdots+\frac{\theta_{1.1}+2(n-1) \pi}{f_{n}}\right)+\delta
$$

By the formula (6), the global main natural frequency is weighted average of the multiple harmonics main natural frequency. Selection of cutoff frequency $f_{n}$ is need to be further determined. From the perspective of energy, assuming $E_{n}$ is the $\mathrm{n}$ times the natural frequency $f_{n}$ of the corresponding energy, must meet the following formula:

$$
\frac{E_{n}}{\sum_{k=1}^{n} E_{k}} \geq \varepsilon
$$




\section{The Algorithm Implementation of Electricity Transmission Line Fault Location Based on Travelling Wave.}

When transmission line failure occurs, three phase current data on both ends of the line using Clarke and Karen Bauer method to decouple, and then select the corresponding modulus as the research object according to the fault type. EEMD ${ }^{[4]}$ decomposition of the modulus, eliminate irrelevant component, WVD transform and overlay each component, solve the problem of WVD crossover study. Calculate the global main natural frequency using the previous section derivation principle and then calculate fault distance. The specific process is shown as figure 2.

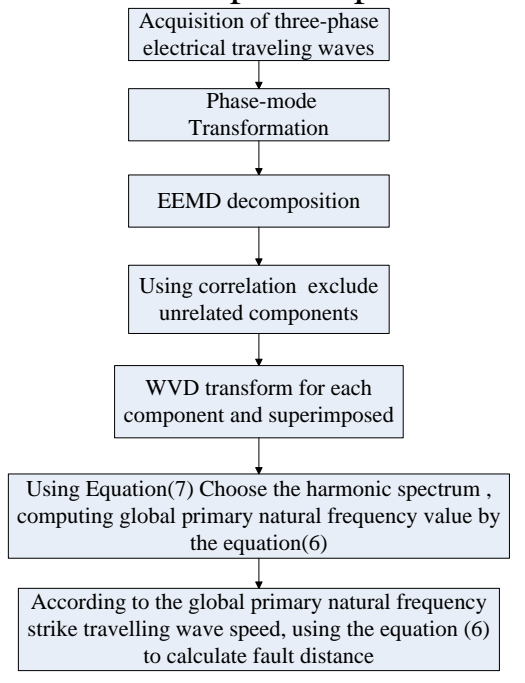

Fig.2 Process of fault location

\section{Simulation and Analysis}

Case Simulation. Build $500 \mathrm{kv}$ transmission line simulation model On PSCAD platform as figure1. Line is 500, of which the power 1: $500 \angle 50 \mathrm{kV}, 495 \angle 30 \mathrm{kV}$, soil resistivity $\mathrm{R}=100 \Omega \cdot \mathrm{m}$, line parameters $r_{1}=8.41 \times 10^{-3} \Omega / \mathrm{km}, x_{1}=0.264 \Omega / \mathrm{km}, b_{1}=4.33 \times 10^{-9} \mathrm{~S} / \mathrm{km}, r_{0}=0.195 \Omega / \mathrm{km}$, $x_{0}=0.687 \Omega / \mathrm{km}, b_{0}=4.33 \times 10^{-9} \mathrm{~S} / \mathrm{km}$, both ends of capacitance is $0.01 \mathrm{uF}$. Failure occurs in $0.1 \mathrm{~s}$, continuous $0.4 \mathrm{~s}$, sampling frequency is $100 \mathrm{KHZ}$, failure occurs in the $300 \mathrm{~km}$ from Busbar 1 end.

In three-phase phase/ground transmission line short-circuit fault, for example, over resistance to $30 \Omega$, obtain fault current in the Busbar 1 end, join the signal-to-noise ratio of 20 white noise, the Busbar 1 side fault current spectrum after the three phase current signal processing is shown in figure 3.

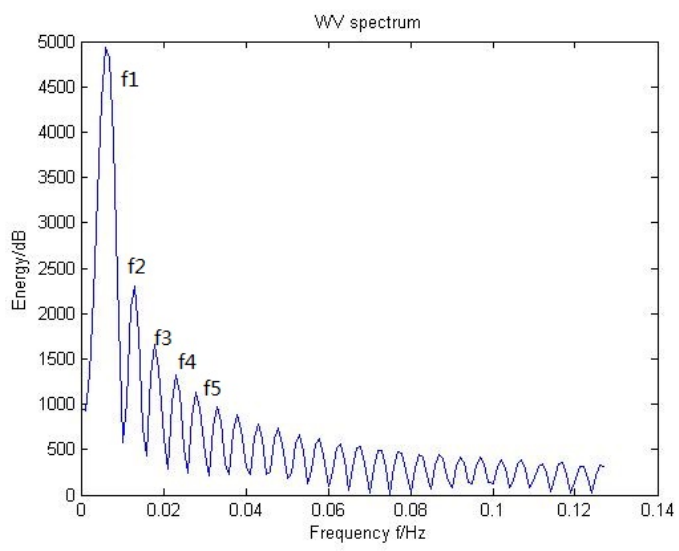

Fig.3 Sepectrum of current in the Busbar 1 side

In the actual selection of frequency, the calculated global advocate natural frequency $f_{\theta}$ is different with the actual value when take $\varepsilon$ to 0.1 , but the error is acceptable. According to the algorithm of 2 shows the process right now, by the frequency to $f_{5}$, from figure 3 spectrum $f_{1}=$ 
$600 \mathrm{~Hz}, f_{2}=1332 \mathrm{~Hz}, f_{3}=1806 \mathrm{~Hz}, f_{4}=2284 \mathrm{~Hz}, f_{5}=2797 \mathrm{~Hz}$, the reflection angle in line parameter 1 side $\theta=4.148$, the distance between fault point and 1 end by the formula (5) (6) is $297.734 \mathrm{~km}$. From table 1: compared with the fault distance considering the main natural frequency multiple harmonics and with the main natural frequency compared to fundamental wave get fault distance, has obvious increase in accuracy.

Tab.1 fault location results before and after

\begin{tabular}{|c|c|c|}
\hline \multirow[t]{2}{*}{ parameters } & $\begin{array}{l}\text { Natural frequency principal } \\
\text { component peak }\end{array}$ & $\begin{array}{c}\text { Natural frequency principal } \\
\text { component multiple } \\
\text { harmonic peaks }\end{array}$ \\
\hline & $f_{1}=600$ & $\begin{array}{c}f_{1}=600, f_{2}=1332, \quad f_{3}=1806, \\
f_{4}=2284, \quad f_{5}=2797\end{array}$ \\
\hline Wave velocity $v /(\mathrm{km} / \mathrm{s})$ & 2.5037 & 2.5037 \\
\hline Reflection angle $\theta /(\mathrm{rad})$ & 4.148 & 4.148 \\
\hline Global natural frequency $f_{\theta}$ & 0.00691 & 0.00747 \\
\hline Measuring distance $/(\mathrm{km})$ & 275.619 & 297.734 \\
\hline Relative error $/(\%)$ & 8.127 & 0.755 \\
\hline
\end{tabular}

Adaptability Analysis and Comparison. Three-phase phase/ground transmission line short-circuit fault occurs in a different location, the algorithm improve error before and after shown in Figure 4. After adding different noise ratio of the white noise to signal, the algorithm improves positioning error changing with the distance of the fault shown in Figure 5. By comparison, considering the results of the fundamental and multiple harmonics locate calculated than considering only the fundamental get more accurate results.

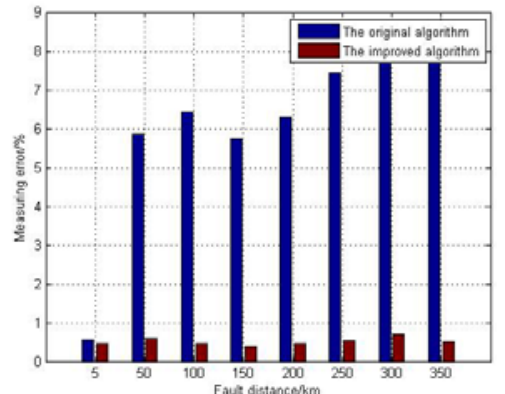

Fig.4 Fault location error of different fault positions

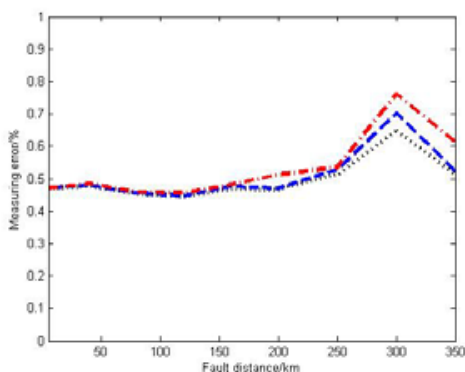

Fig.5 Fault location results of different noise level

\section{Summary}

Based on the positioning of the main natural frequencies ,this paper have been made improvement on the determination of main natural frequencies, considering the fundamental and multiple harmonics to solve the problem that rely on a single frequency positioning is instable, the ranging accuracy has been improved. While on the $500 \mathrm{KV}$ dual power transmission system simulation model, by running at different distances and different SNR conditions, confirming the fault location algorithm of this article has high accuracy, good reliability, error range can be accepted.

\section{References}

[1] Swift G W. The spectra of fault-induced transients[J]. IEEE Transaction on Power Apparatus and Systems, 1979, 98(3): 940-947

[2] Wu Linyong, HE Zhengyou, QIAN Qingquan. A frequency domain approach to single-ended traveling wave fault location [J]. Proceedings of the CSEE, 2008, 28(25): 99-104 
[3] LIN Sheng, WU Xiao, HE Zhengyou, QIAN Qingquan. A power system fault location method based on natural frequency of travelling waves[J]. Power System Technology, 2013, 37(1): 270-275.

[4] ZHU Ninhui, BAI Xiaoming, DONG Weijie. Harmonic detection method based on EEMD[J]. Processing of the CSEE, 2013, 33(7): 14, 92-98. 